\title{
Remote Sensing Image Based Analysis on Present Land Utilization of Chongqing Area
}

\author{
Chanjuan Li \\ Department of Military Medical Geography, Third Military Medical University \\ Chongqing 400038, China \\ Key Laboratory of High Altitude Medicine (Third Military Medical University) \\ Ministry of Education, Chongqing 400038, China \\ Tel: 86-1301-2358-530 E-mail: yocatg@163.com
}

\begin{abstract}
Based on the remote sensing images taken by China-Brazil earth resource satellite during July 2006, the paper describes eight types of land utilization of 1,612 km2 city Chongqing area. As a result, the actual area and corresponding proportion of each type of land utilization is obtained. In addition, the paper has analyzed current land utilization status of the area.
\end{abstract}

Keywords: City Chongqing, Remote sensing interpretation, Present land utilization

\section{Introduction}

Remote sensing interpretation can be characterized as an inverse procedure of remote sensing imaging. It reconstructs a geographic landscape through target recognition and phenomenon identification, which is in turn achieved by analyzing a variety of characteristic information provided by the images acquired through remote sensing. Based on the remote sensing images, this paper analyzes plant coverage across major area of Chongqing. Chongqing is a well known city in mountainous area with complex terrain condition. The complexity of mountainous terrain makes it difficult to identify the information from remote sensing images, resulting in a relatively false conclusion from image interpretation. At present, there are mainly two types of interpretation methodologies: visual interpretation and computerized digital pattern recognition. Although visual interpretation is easy and feasible, it can not process large amount information in a quantitative way. Computerized digital pattern recognition generally takes an approach of interactive effort from both user and computer, is able to process in qualitative and quantitative analysis. However, process speed and quality of results are directly impacted by algorithm. In addition, the results are largely based on statistical modeling, are hardly precise in describing a specific and real geographic terrain.

\section{Outline of the studied area and data source}

The studied area includes Chongqing city and surrounding area, a total of 1,612 $\mathrm{km} 2$. The area also includes Jinyunshan, Geleshan and part of Nanshan. Chongqing city is located near to where Changjiang River meets Jialingjing River. Surrounded by mountains and embraced by rivers, the water front city is built on numerous hills and valleys, known by its water and famous by its picturesque view. Geographically, the terrain is mainly 
complex and diversified mountainous area.

Basic data used for this study comes from the remote sensing images taken by China-Brazil earth resource satellite during July 2006. The images studied were taken by IRMSS (Infrared Multi-Spectral Scanner). The spatial resolution of the images is $20 \mathrm{~m}$. Images have 4 bands of spectrum, namely 1, 2, 3, and 4 . The study chose 2, 3, and 4 bands of spectrum designated with red, blue and green. The reference data are based on 1:50,000 topographic map and 1:200,000 present land utilization map of Chongqing.

\section{Research methodology and pre-processing of the remote sensing images}

\subsection{Research methodology}

There are many different approaches to interpret remote sensing image, including frequency domain analysis with high resolution image, texture processing, high resolution SSMC, interactive method between researcher and computer, neural network, etc. (Zhang, Su, 2008; Yang, Bin, 2004; Huang, Xin, 2006; Zhang, Songling, 1999; Zhou, Tinggang, 2004). The most critical part of remote sensing interpretation is image processing and classification.

The primary study methodology includes comprehensive applications of unsupervised classification and supervised classification, cluster analysis, filtration analysis, depletion analysis and et al (Dang, Anrong, 2004, p. 186-237) on the selected remote sensing images to achieve the interpretation and classification of Chongqing city area. The preliminary process of the images includes geometric correction, boundary tracking, radiometric correction and et al. The remote sensing images were taken by China-Brazil earth resources satellite during July 2006. The image processing tool and software include Erdas Imagine 8.7, Arc GIS 9.0 and GTS.

\subsection{Remote sensing data processing}

The remote sensing images inevitably have statistical and systematic deformation errors. Images may exhibit one-on-many object-spectrum or many-on-one object-spectrum mapping. In addition, seasonal change may affect the consistency of the images. Preliminary image process is necessary before image interpretation, which includes image projection and coordinate transformation, dissection of the studied area, topographic reconstruction, precision geometric correction, artificial color composition and et al. Figure 1 shows the processed remote sensing image.

$<$ Figure $1>$

3.2.1 Data format conversion and coordinate transformation

The data, provided by the ground station for satellite remote sensing at the Chinese Academy of Sciences, is given in BSQ format. Data of each band is independently saved as a binary grayscale image file and a header file. The header file includes variety of information for interpreting the image. Using coordinate data at four corners in the header file, one can transfer the format of remote sensing data from matrix coordinate system into real space Gauss coordinate system.

\subsubsection{Precision geometric correction}

Remote sensing data is processed with systematic correction. It has a high degree of geometric deviation. It is necessary to process the data with precision geometric correction. Taking the gully line and ridge line from a 1:50,000 topographic map as the precision geometric correction reference in the control layer. Sufficient correction control point can be obtained and the geometric error can be corrected to the limit of one pixel.

\subsubsection{Image extraction of the studied area}

The studied area is extracted from 1:50,000 topographic map. With buffer analysis, the area is determined by 
expanding the administrative boundary outward by $1.5 \mathrm{~km}$ in order to keep the data integrity of the studied area after precision geometric correction.

\subsubsection{Elimination of orographic influence}

Topographic variation and sun elevation angle often introduces aberrant spectrum. To keep the interpretation and analysis accurate, the same forest vegetation at different slope direction or different slope position are modeled with the same spectral parameters, therefore eliminates the orographic influence.

\section{Interpretation and classification of remote sensing image}

\subsection{Classification of land utilization}

Since the study is based on the quality change of ecological environment, the type of land utilization of the city Chongqing is classified into dry land, paddy field, forest land, shrub land, unutilized land, river, lake and urban construction land according to relate ecological safety assessment standard (Xie, Hualin, 2004; Gong, Jianzhou, 2006; Zuo, Wei, 2003).

As for unsupervised classification, we classify the land utilization into 24 types, more accurate and more detailed than above-mentioned classification.

\subsection{Comprehensive interpretation with data on geographical environment}

Complex topography and geomorphology will influence the interpretation of image. It is essential to investigate the data about geographic environment of the studied area comprehensively. Aberrant spectrum, occurred in interpreting forest vegetation, will lead to different vegetation types with identical spectral characteristics. It is the most basic interpretation procedure to use the data on habitat condition and environmental gradient as auxiliary data, such as altitude, slope direction, slope position, slope gradient, soil and et al. The study quantify the habitat data by spatial overlay analysis method; then directly calculate at each map spot of vector data; finally, interpret the image by man-machine interactive process with above-mentioned data. As a result, the interpretation accuracy and work efficiency are improved significantly.

\subsection{Establishment of interpretation criteria}

Automatically interpret the remote sensing image by unsupervised classification to obtain the interpretation criteria; then revise and merge these interpretation criteria by visual interpretation with reference to previous investigations on forest, land utilization, present land-utilization graph and et al of Chongqing. A detailed and accurate interpretation criterion is finally established.

\subsection{Classification}

First, get the interpretation criteria with unsupervised classification process; then process the treated remote sensing image by supervised classification process with the revised interpretation criteria. Land-utilization types are classified into 9 classes. Process these classes with cluster analysis, filter analysis and depletion analysis; Recode the obtained types (Table 1 shows the recoded types). After recoding, transfer the interpreted grid map into editable vectorgraph by Erdas software; establish the topological relation among elements of the map layers by cleaning and building vectorgraph; introduce the obtained data with Arcinfo format into Arcmap, eliminate or merge the map smaller than $5,000 \mathrm{~m} 2$ by Arcmap. As a result, data is reduced by about $30 \%$ so that the further operation is more convenient.

$<$ Table 1>

\subsection{Edit of interpretation data}

In Arcmap, open the automatically generated vector data and integrated environmental gradient data, establish the interpretation criteria. Data directly interpreted by Erdas is different from the actual conditions; therefore it 
is essential to revise the obvious defects in the map by comparing the original remote sensing image to the present land-utilization map. Based on the remote sensing algorithm, color codes of ground objects and environmental gradient data in the attribute database of vectorgraph, interpret the attribute of terrestrial objects in batch and write them into the attribute database of vectorgraph. Verify the interpreted results combined with ecological distribution and revise the batch processing conditions. According to the interpreted land-utilization types in 2006, integrate the spatially connected land of the same category to get the map spot layers. With this approach, we obtain the data of the picture spots in city Chongqing in 2006 from above-mentioned map spot layer. Figure 2 is the interpretation graph of remote sensing image of Chongqing area.

$<$ Figure 2>

\section{Interpretation and classification of remote sensing image}

Table 2 shows all 8 types of land-utilization types in city Chongqing. Vegetation coverage is about $50 \%$ (forest land accounts for $10.2 \%$ and shrub land accounts for $37.74 \%$ ); urban construction land (the city and nearby suburban area) is $20.40 \%$; dry land is $23.84 \%$; paddy field is $2.60 \%$; river is $3.36 \%$; unutilized land is $0.97 \%$. Among them, there is vast area of river because the center part of city is at the junction of Changjiang River and Jialingjiang River. In addition, forest land is also large, one reason is the strong protection for the original forest land; the other is increased conversion of cropland to forest and artificial afforestation. However, paddy field is small, this is because that Chongqing is a mountain city lack of paddy field; also, most paddy fields turn into dry land because of water shortage. Meanwhile, many paddy fields are occupied with the rapid development of city.

$<$ Table 2>

\section{Conclusion}

Remote sensing interpretation method is an accurate and effective procedure to collect data on the land utilization. Based on the remote sensing images taken by China-Brazil earth resources satellite in July 2006, with preliminary correction and processing, then interpretation and classification to get vector data, finally introduction them into Arcmap to edit and revise, we acquired the present land-utilization data in city Chongqing. In the entire process, the more difficult parts are preliminary process of original data and revision of vector data. Both aspects need to refer relevant data. With repeated corrections, good results could be achieved. However, there are still room for improvement in the process and results. First of all, geographical environment has effects on the data since same object may have different spectrums and different objects may have same spectrum in the original images. Although many scholars have developed some effective methodologies, these methods still have their limitations, especially facing mountainous terrain, that effectiveness is limited. Secondly, the amount of vector data is large and difficult to process. Although they can be merged to certain degree, they are relying on manual process and efficiency is low. These two issues should be addressed in the future study.

\section{References}

Cao, Yu, Chen, Hui, Ouyang, Hua \& Xiao Duning. (2006). Landscape ecological classification using vegetation indices based on remote sensing data: a case study of ejin natural oasis landscape. Journal of Natural Resources, 21(3), 481-488.

Dang, Anrong \& Wang, Xiaodong. (2004). Remote sensing image treatment by ERDAS IMAGINE. Beijing: Tsinghua University Press. p. 186-237.

Gong, Jianzhou, Xia, Beicheng \& Guo, Luo. (2006). Assessment and prediction models of urban ecological 
security. Acta Scientiarum Naturalium Universitatis Sunyatseni, 45(1), 107-111.

Huang, Xin, Zhang, Liangpei \& Li, Pingxiang. (2006). SSMC method for the classification of high spatial resolution remote sensing images. Journal of Image and Graphics, 11(4), 529-534.

Xie, Hualin \& Li, Bo. (2004). A study on indices system and assessment criterion of ecological security for city. Journal of Beijing Normal University (Natural Science), 40(5), 705-710.

Yang, Bin, Zhao, Hongman, Zhao, Zongtao \& Zhang Le. (2004). An improved texture classification and distinction algorithm for remote sensing object. Microelectronics \& Computer, 21(9), 1111-1123.

Zhang, Songling, Yang, Bangjie, Wang, Fei \& Pei, Zhiyuan. (1999). An interactive image interpreting system for landuse remote sensing monitoring based on GIS. Transactions of the Chinese Society of Agricultural Engineering, 15(2), 185-188.

Zhang, Su. (2008). The research on the application of geographic frequency spectroscopy in interpreting remote sensing image. Journal of Chengdu University of Technology (Science \& Technology Edition), 33(2), 198-202.

Zhou, Tinggang \& Su, Yingchun. (2004). Artificial neural networks-based study of vegetations classification for aerial remote sensing image. Journal of Southwest China Normal University (Natural Science), 29(6), 1037-1040.

Zuo, Wei, Zhou, Huizhen \& Wang, Qiao. (2003). Conceptual framework for selection of an indicator system for assessment of regional ecological safety. Soils, 1, 2-7.

Table 1. Classification of remote sensing interpretation criteria of land-utilization types in city Chongqing

\begin{tabular}{|c|c|c|c|c|c|c|c|c|}
\hline $\begin{array}{c}\text { Land-utilization } \\
\text { types }\end{array}$ & $\begin{array}{c}\text { Dry } \\
\text { land }\end{array}$ & $\begin{array}{c}\text { Paddy } \\
\text { field }\end{array}$ & $\begin{array}{c}\text { Forest } \\
\text { land }\end{array}$ & $\begin{array}{c}\text { Shrub } \\
\text { land }\end{array}$ & $\begin{array}{c}\text { Unutilized } \\
\text { land }\end{array}$ & River & Lake & $\begin{array}{c}\text { Urban } \\
\text { Construction land }\end{array}$ \\
\hline Serial number & 1 & 2 & 3 & 4 & 5 & 6 & 7 & 8 \\
\hline
\end{tabular}

Table 2. Present land utilization in city Chongqing

\begin{tabular}{|c|c|c|c|c|c|c|c|c|}
\hline $\begin{array}{c}\text { Land-utilization } \\
\text { types }\end{array}$ & $\begin{array}{c}\text { Urban } \\
\text { construction } \\
\text { land }\end{array}$ & Dry land & $\begin{array}{c}\text { Forest } \\
\text { land }\end{array}$ & $\begin{array}{c}\text { Shrub } \\
\text { land }\end{array}$ & $\begin{array}{c}\text { Reservoi } \\
\mathrm{r}\end{array}$ & Paddy & River & $\begin{array}{c}\text { Unutilize } \\
\mathrm{d} \text { land }\end{array}$ \\
\hline Area $\left(\mathrm{km}^{2}\right)$ & 329.0695 & 384.5616 & 164.5483 & 608.7176 & 14.3714 & $\begin{array}{c}41.954 \\
2\end{array}$ & $\begin{array}{c}54.209 \\
3\end{array}$ & 15.3644 \\
\hline Proportion(\%) & 20.40 & 23.84 & 10.20 & 37.74 & 0.89 & 2.60 & 3.36 & 0.97 \\
\hline
\end{tabular}




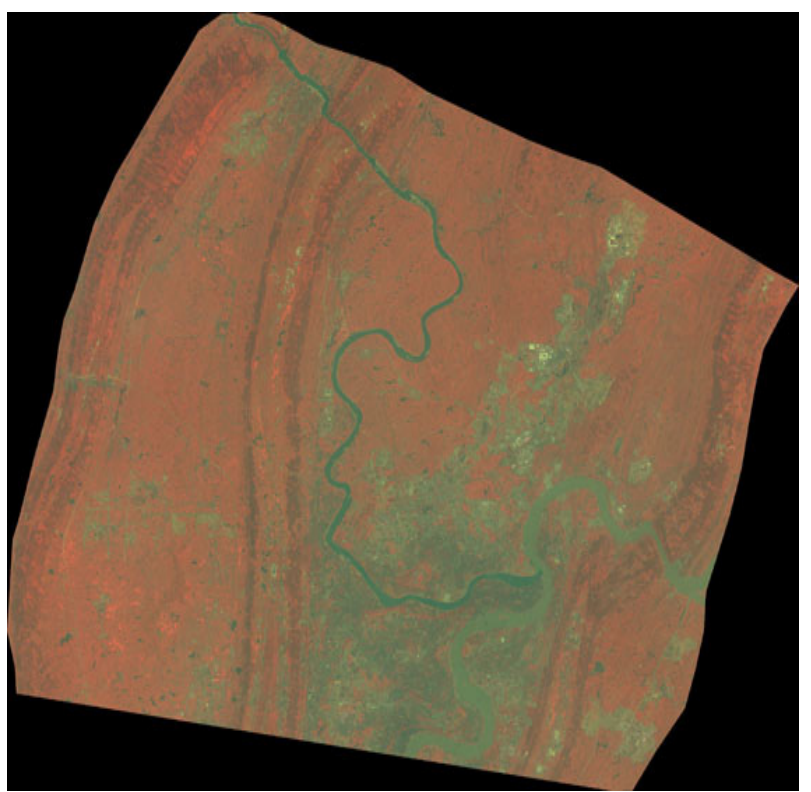

Figure 1. The Processed Remote Sensing Image

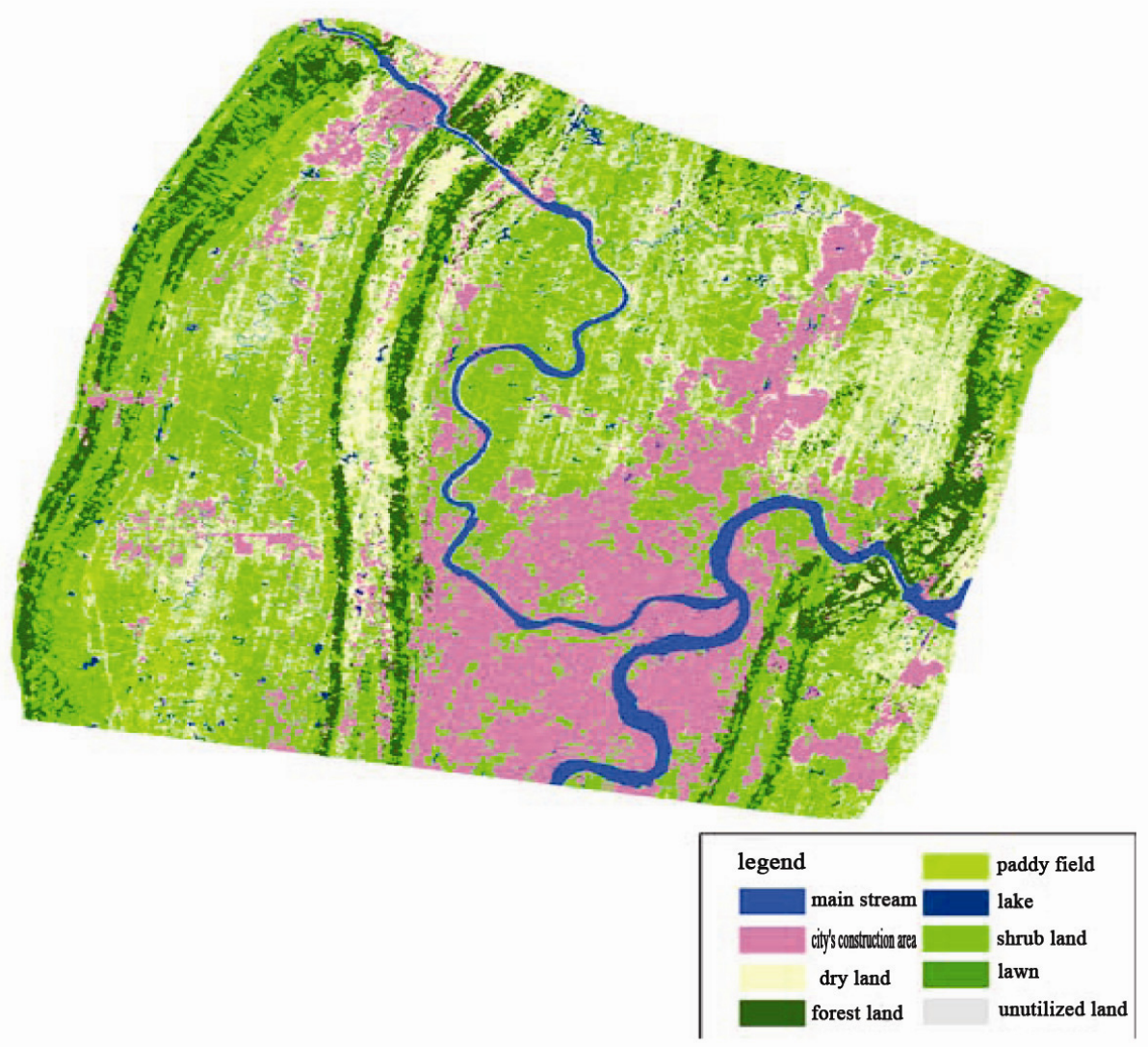

Figure 2. The Interpretation Graph of Remote Sensing Image of Chongqing 